Check for updates

Cite this: Soft Matter, 2020, 16,4267

Received 4th February 2020, Accepted 3rd April 2020

DOI: 10.1039/d0sm00208a

rsc.li/soft-matter-journal

\section{Ordering of binary colloidal crystals by random potentials $\dagger$}

\author{
André S. Nunes, ${ }^{* a}$ Sabareesh K. P. Velu, (D) $*^{\mathrm{b}}$ Iryna Kasianiuk, ${ }^{\mathrm{c}}$ Denis Kasyanyuk, ${ }^{\mathrm{c}}$ \\ Agnese Callegari, (D) C Giorgio Volpe, (D) ${ }^{\mathrm{d}}$ Margarida M. Telo da Gama, (D) ${ }^{\mathrm{a}}$ \\ Giovanni Volpe (D) be and Nuno A. M. Araújo (D) $\ddagger^{a}$
}

\begin{abstract}
Structural defects are ubiquitous in condensed matter, and not always a nuisance. For example, they underlie phenomena such as Anderson localization and hyperuniformity, and they are now being exploited to engineer novel materials. Here, we show experimentally that the density of structural defects in a 2D binary colloidal crystal can be engineered with a random potential. We generate the random potential using an optical speckle pattern, whose induced forces act strongly on one species of particles (strong particles) and weakly on the other (weak particles). Thus, the strong particles are more attracted to the randomly distributed local minima of the optical potential, leaving a trail of defects in the crystalline structure of the colloidal crystal. While, as expected, the crystalline ordering initially decreases with an increasing fraction of strong particles, the crystalline order is surprisingly recovered for sufficiently large fractions. We confirm our experimental results with particle-based simulations, which permit us to elucidate how this non-monotonic behavior results from the competition between the particle-potential and particle-particle interactions.
\end{abstract}

\section{Introduction}

Perfect crystalline structures are not commonly found in Nature, because, even in the absence of impurities, structural defects occur spontaneously and disrupt the periodicity of the crystalline lattice. ${ }^{1}$ For example, when a melt is cooled down, multiple crystallites grow with degenerate orientations. ${ }^{2}$ Since the coarsening time of these crystallites diverges with size, structural defects appear and prevent the emergence of global order., While the existence of these defects is a challenge when growing single crystals, it can also be an opportunity when engineering the properties of materials; indeed, control over defects enables the development of solid-state devices with fine-tuned mechanical resilience, optical properties, and heat and electrical conductivity. ${ }^{5-9}$ In atomic crystals, engineering structural defects is an experimental challenge for two reasons: ${ }^{10}$

${ }^{a}$ Centro de Física Teórica e Computacional and Departamento de Física, Faculdade de Ciências, Universidade de Lisboa, P-1749-016 Lisboa, Portugal. E-mail:nmaraujo@fc.ul.pt

${ }^{b}$ Department of Physics, Bilkent University, Cankaya, 06800 Ankara, Turkey

${ }^{c}$ Department of Physics, Bilkent University and UNAM, Cankaya, 06800 Ankara, Turkey

${ }^{d}$ Department of Chemistry, University College London, 20 Gordon Street, London WC1H OAJ, UK

${ }^{e}$ Department of Physics, University of Gothenburg, 41296 Gothenburg, Sweden

$\dagger$ Electronic supplementary information (ESI) available. See DOI: 10.1039/d0sm00208a

$\ddagger$ Contributed equally. first, current visualization techniques at the atomic scale do not provide a high spatial or time resolution; ${ }^{11,12}$ second, no current technique can control the density of defects in a systematic manner. ${ }^{13}$ The first challenge can be overcome studying colloidal crystals as models for atomic systems, ${ }^{14,15}$ where colloidal particles can be individually tracked using standard digital video microscopy techniques, ${ }^{16-18}$ and have in fact also been used to study crystallisation and melting of colloidal crystals in the presence of extended laser fields. ${ }^{19,20}$ Here, we demonstrate that the second challenge can be solved combining a binary colloidal mixture and an optical random potential generated by a speckle light pattern. This permits us to control the density of structural defects in the resulting $2 \mathrm{D}$ colloidal crystal and to explore a surprising non-monotonic behavior of their ordering and stability.

\section{Results}

We use a binary colloidal suspension of equally-sized polystyrene (refractive index $\left.n_{\mathrm{PS}} \approx 1.59\right)$ and silica $\left(n_{\mathrm{Si}} \approx 1.42\right)$ spherical particles with diameters $d_{\mathrm{PS}}=6.24 \pm 0.22 \mu \mathrm{m}$ and $d_{\mathrm{Si}}=6.73 \pm 0.22 \mu \mathrm{m}$, respectively. The particles interactions are hard-sphere like but the following results can be reproduced with soft interactions as well (see ESI $\dagger$ ). ${ }^{21}$ To characterize the composition of the mixture, we use the molar fraction of polystyrene particles defined as $\chi=N_{\mathrm{ps}} / N_{\mathrm{t}}$ where $N_{\mathrm{ps}}$ is the 
number of polystyrene particles and $N_{\mathrm{t}}$ is the total number of particles. We let these particles sediment at the bottom surface of a homemade sample chamber so that they are effectively confined in a quasi-2D space (see the Section Materials and methods). We illuminate from above with a speckle pattern, which we generate by mode-mixing a laser beam in a multimode optical fibre (see Fig. 5). ${ }^{22-24}$ Speckle patterns form rough, disordered optical potentials characterized by wells whose depths are exponentially distributed, with spatial correlations that are Gaussian with an average width (grain size) set by diffraction. As proposed in ref. 25, to characterize the strength and correlation length of the optically generated random field, we first identify the "bright spots" and then fit a Gaussian to each spot, using the code in ref. 26. We found $\sigma=2.7 \pm 0.2 \mu \mathrm{m}$, which is less than half the diameter of the particles. Furthermore, the fibre imposes a Gaussian envelope (beam waist $\sigma_{\mathrm{G}}=72.5 \pm 0.2 \mu \mathrm{m}$ ) to the speckle pattern, which attracts the particles towards the center of the speckle pattern effectively confining them in space. Since the optical forces acting on the particles increase for larger mismatches between their refractive index and that of the surrounding medium (here water, $n_{\mathrm{w}} \approx 1.33$ ), ${ }^{27}$ the optical forces acting on the polystyrene (strong) particles are about $2 \times$ higher than those exerted on silica (weak) particles (estimated using the FORMA $\operatorname{method}^{28}$ ). Importantly, the optical forces at the deepest local minima of the speckle potential are strong enough to trap the strong particles, but not the weak ones (see $\mathrm{ESI} \dagger$ for an estimation of the strength of the optical traps ${ }^{21}$ ).

We start with a low concentration of particles $\left(1.4 \times 10^{7} \mathrm{ml}^{-1}\right)$ and switch on the optical potential. The particles are attracted towards its center by the Gaussian envelope. When only weak particles are present $(\chi=0)$, they eventually form a compact structure with hexagonal order, as shown in Fig. 1a. When we introduce strong particles, these get trapped in the local minima of

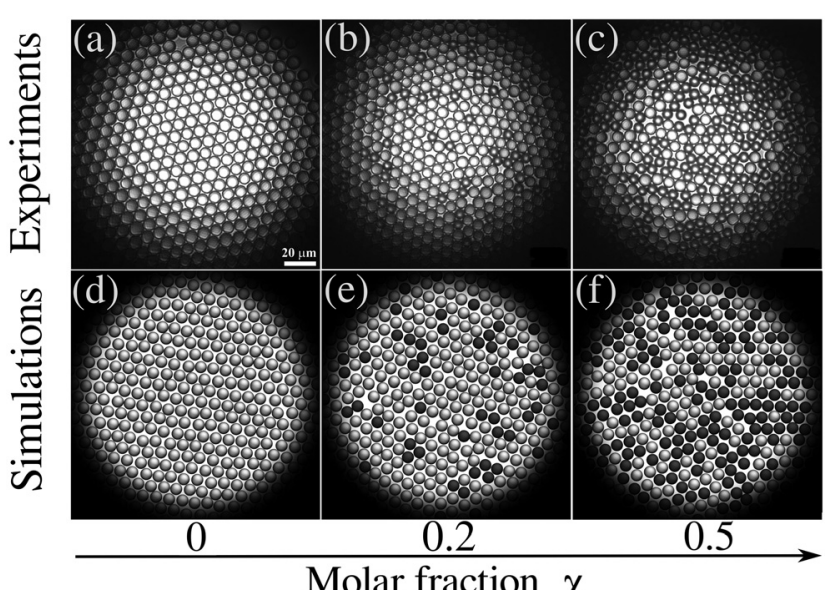

Fig. 1 Colloidal crystals with tunable degree of disorder. Final configurations obtained in $(a-c)$ experiments and $(d-f)$ simulations, for different molar fractions $\chi$ of strong particles. The weak (silica) particles are light gray, and the strong (polystyrene) particles are dark gray. The illumination for the images is delivered by an optical fibre which produces the vignetting effect observed in the experimental images. the disordered potential and introduce defects that reduce the hexagonal order. Already with only $20 \%$ of strong particles $(\chi=0.2)$, the presence of structural defects is clearly visible (see Fig. 1b). The impact is even more pronounced when $50 \%$ of the particles $(\chi=0.5)$ are strongly interacting with the potential (Fig. 1c). Thus, strong particles act as defects in the crystalline structure of the weak ones, compromising global order. We were able to determine that the deformation of the structure was not caused by particle bidispersity since when subject only to a Gaussian envelope the particles formed a crystalline structure independently of the number of strong particles present (see ESI $\dagger$ ). ${ }^{21}$ The experimental results are confirmed by particle-based simulations, as shown in Fig. 1d-f (see Section Materials and methods). As we will see in more detail below, we can control the density of defects by adjusting $\chi$ as well as the intensity and grain size of the pattern.

To quantify the order of the crystalline structure, we measure the six-fold bond-order parameter, $\left\langle\phi_{6}\right\rangle$, defined as ${ }^{29}$

$$
\left\langle\phi_{6}\right\rangle=\frac{1}{6 N_{\mathrm{c}}} \sum_{l}^{N_{\mathrm{c}}}\left|\sum_{j}^{N_{\mathrm{b}}} \mathrm{e}^{i 6 \theta_{l j}}\right|,
$$

where the out sum is over the $N_{\mathrm{c}}$ particles within 7.5 particle diameters from the center of the potential (the area shown in Fig. 1), which is the area where the aggregate is formed and does not include the boundary particles. The inner sum is over the $N_{\mathrm{b}}$ neighbors of a particle in the Voronoi tessellation, and $\theta_{l j}$ is the angle between the $x$-axis and the line connecting the centers of particles $j$ and $l .\left\langle\phi_{6}\right\rangle=1$ for perfect hexagonal crystals (in practice, it is never exactly one, because of thermal fluctuations and other transient perturbations to the periodic order) and it decreases with the number of structural defects. Fig. 2 shows $\left\langle\phi_{6}\right\rangle$ obtained experimentally and numerically as a function of the molar fraction $\chi$. For $\chi=0,\left\langle\phi_{6}\right\rangle \approx 1$, consistent with the formation of an hexagonal periodic structure. As expected, as $\chi$ increases, the value of $\left\langle\phi_{6}\right\rangle$ decreases due to the formation of structural defects. The snapshots in the top rows of Fig. 2 show the final configurations (first row), the corresponding Voronoi tessellations (second row), and the spatial Fourier transform (third row), for different values of $\chi$.

Surprisingly, the data reported in Fig. 2 show that $\left\langle\phi_{6}\right\rangle$ reaches a minimum at $\chi_{\min } \approx 0.6$, and that the global order increases for $\chi>\chi_{\text {min }}$. In particular, for $\chi=1$, the strong particles self-assemble into a hexagonal crystal, despite the presence of the underlying random potential. This nonmonotonic dependence is also observed at higher densities. In Fig. S5 of the ESI, $\dagger^{21}$ we shown that the same behavior is observed numerically in a system with a number of particles that is $25 \%$ higher. This result is corroborated by the Voronoi tessellation of the final configurations and by the respective spatial Fourier transforms. From this analysis, we can see that the number of Voronoi cells with a number of neighbors different from six becomes higher near the minimum of $\left\langle\phi_{6}\right\rangle$, even though the Voronoi-cell size in both experiments and simulations does not vary significantly compared with the particle size (see Fig. S6 from the ESI $\dagger$ ). ${ }^{21}$ Also, The Fourier 
(i) $\chi=0$

(ii) $\chi=0.23$

(iii) $\chi=0.6$

(iv) $\chi=1$
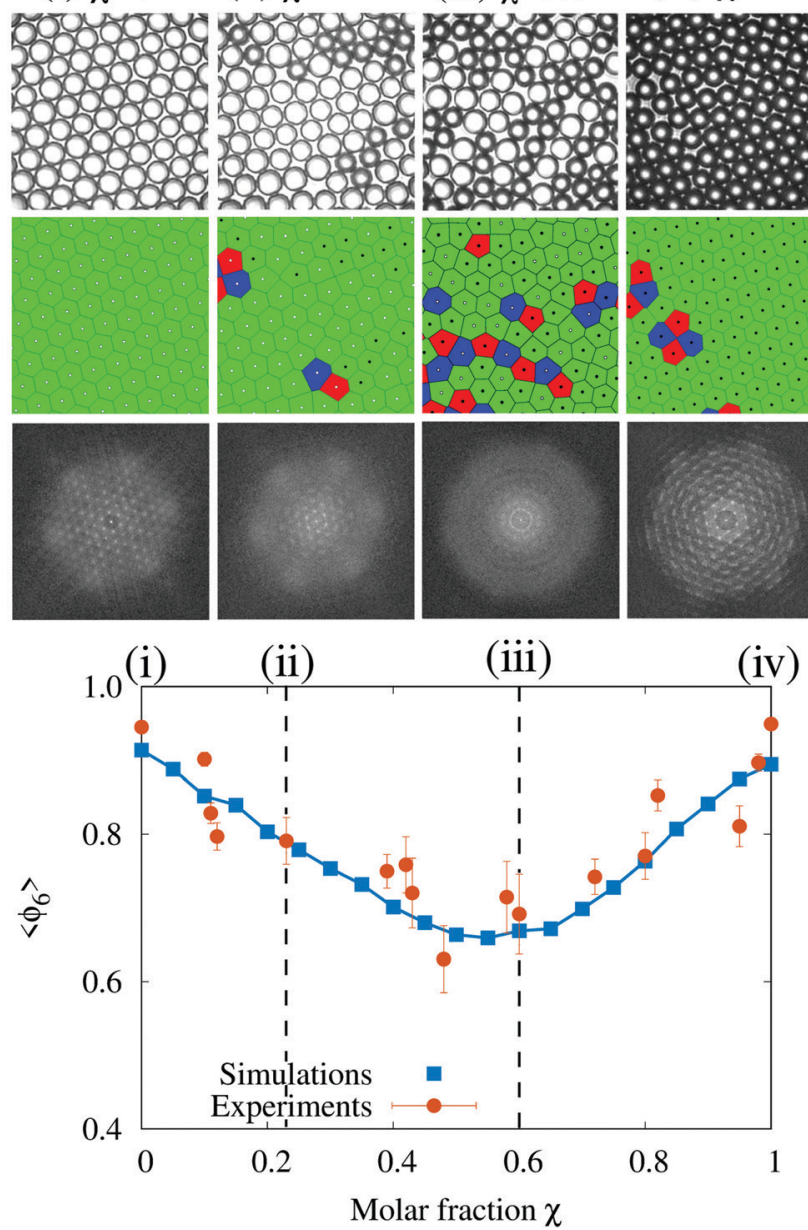

Fig. 2 Crystalline order for different molar fractions of strong particles. Six-fold bond order parameter $\left\langle\phi_{6}\right\rangle$ as a function of the molar fraction $\chi$ obtained experimentally (circles) and numerically (squares; the blue line connects the symbols for visual guidance). The error bars show the standard deviation of $\left\langle\phi_{6}\right\rangle$ over 500 frames in the stationary state of the experiments (i.e., after 30 minutes from the start of the experiments). The numerical results are averages over 100 samples. The top snapshots show the final configurations in the experiments (first row), the Voronoi tessellation (second row), and the spatial Fourier transform (third row) for $\chi=0,0.23,0.6$, and 1 . The filled (empty) circles at the center of the Voronoi cells indicate strong (weak) particles. The cells are colored by the number of nearest neighbors, namely, equal (green), lower (red), greater (blue) than six. See also Supplementary Video 1 (ESI†).

transforms display dimmer intensity peaks near the minimum of $\left\langle\phi_{6}\right\rangle$.

In order to shed light on the non-monotonic behavior, we first analyze the trajectories obtained by particle-based simulations, without the Gaussian envelope and a particle density $10 \times$ lower than that of maximal packing, to study the interactions between the two particle species and the local minima in the potential. Fig. 3(a) shows individual trajectories of weak (light gray) and strong (dark gray) particles at various $\chi$. In all cases, the weak particles can hop between minima, while the strong particles are readily trapped in them. This qualitative analysis for a lower density elucidates the possible underlying mechanisms at higher densities. In the presence of the Gaussian envelope, particles are dragged to the center and the strong particles quickly populate the minima that are sufficiently deep to prevent their escape. At low $\chi$, the number of strong particles is lower than the number of such minima so they remain there for the entire simulation time, because this configuration is energetically favorable (Fig. 3b and c); therefore, the number of spatial defects increases monotonically with the number of the trapped strong particles, leading to a decrease of $\left\langle\phi_{6}\right\rangle$ with increasing $\chi$. At large $\chi$, the number of strong particles is greater than the number of potential minima and thus it becomes energetically favorable to have more than one strong particle in one minimum (Fig. 3d). This allows the spatial rearrangement of the particles since the energy of the interaction with the speckle is no longer strong enough to localize the particles, a large-scale crystalline structure is favorable, consistent with the increase in $\left\langle\phi_{6}\right\rangle$ observed in Fig. 2. When $\chi=1$, all particles are strong and thus the hexagonal crystalline structure is recovered. We also counted the number of strong and weak particles situated in minima of the random potential as a function of $\chi$. As shown in Fig. S8 of the ESI, $\dagger^{21}$ the minima are mainly populated by strong particles and the average number of particles is larger than one for values of $\chi$ above the one at which the six-fold bond order parameter is the minimum.

In order to explore how robust the non-monotonic dependence of $\left\langle\phi_{6}\right\rangle$ as a function of $\chi$ is, we studied numerically how it depends on the properties of the underlying speckle pattern. The speckle is characterized by a strength $V$ corresponding to the average potential depth (in units of $k_{\mathrm{B}} T$, where $k_{\mathrm{B}}$ is the Boltzmann constant and $T$ is the absolute temperature of the sample) and by a spatial correlation $\sigma$ (in units of the particle diameter), which corresponds to the average grain size. Fig. 4(a) shows $\left\langle\phi_{6}\right\rangle$ for different $V$. Although the curves in the range $1.51<V \leq 18.8$ feature one minimum, its position and intensity vary with $V$ : the number of minima that can trap particles is expected to increase with $V$. Thus, the fraction of particles that can be trapped also increases and the corresponding value of $\chi_{\min }$ shifts to the right while the minimum becomes deeper. For $V>18.8$, the behavior seems to become independent of the molar fraction (and always disordered), because the weak particles are also strongly trapped. Fig. 4(b) shows $\left\langle\phi_{6}\right\rangle$ for different values of $\sigma$. A pronounced minimum is only observed for intermediate values of $\sigma$, close to unity (particle diameter). If $\sigma \gg 0.5$ or $\sigma \ll 0.5$, the optical forces are negligible for different reasons: for $\sigma \gg 0.5$, the gradient of the optical potential is very small on the scale of the particle; and for $\sigma \ll 0.5$, the optical potential varies on a length scale smaller than the particle size and thus its gradient averages to zero over the particle cross-section (see Fig. S9, ESI $\dagger$ ). ${ }^{21}$ In the latter case, the optical force on a particle is the sum of the contributions over the particle's cross-section, which can be described by an effective random potential that differs from the one originally applied (Fig. S10 and S11, ESI $\dagger$ ). ${ }^{21}$

\section{Discussion}

In conclusion, we have shown that the order in a twodimensional binary colloidal crystal can be controlled by an 
(a) $\quad x=0.0$

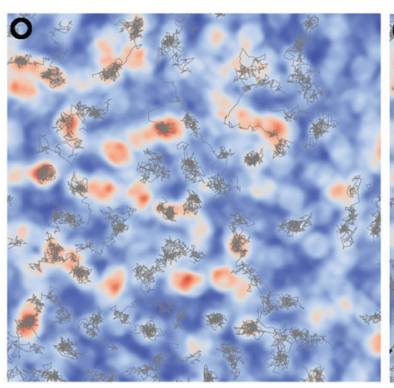

(b)

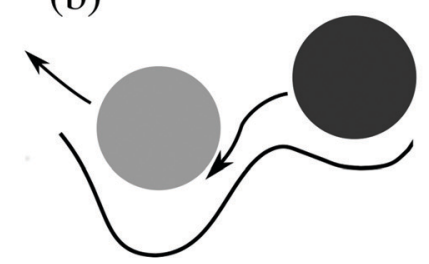

$\chi=0.1$

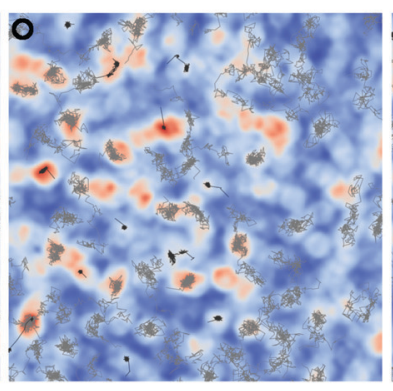

(c)

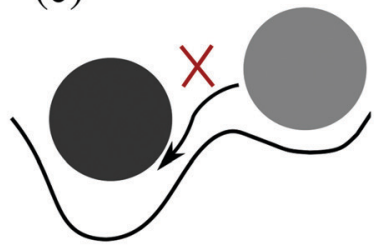

$\chi=0.5$

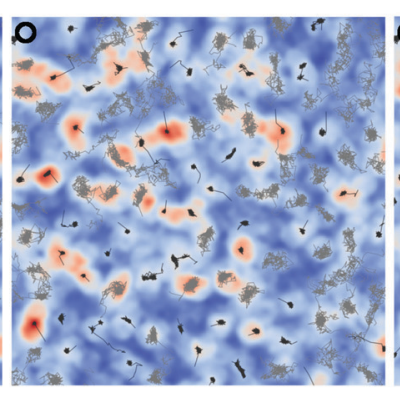

(d)

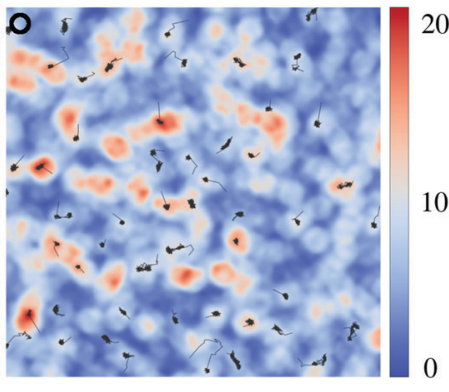

20

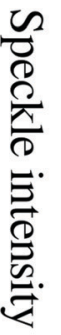

Fig. 3 Local dynamics of the interaction between particles and minima in the random potential. (a) Examples of trajectories of weak (light gray) and strong (dark gray) particles in the presence of a speckle obtained numerically for different values of the molar fraction $\chi$. The particle density is $10 \times$ lower than that of maximal packing and the Gaussian envelope is absent. The four simulations were preformed under exactly the same conditions, including the same sequence of random numbers for the thermostat (see ESI $\dagger$ ) ${ }^{21}$ The black circles on the top left corner indicate the particle size. The random potential intensities are in units of $k_{\mathrm{B}} T$ and $\sigma$ is one particle diameter. (b) When a weak particle (light gray) is located at a potential minimum and a strong particle (dark gray) is in its vicinity, it is energetically favorable to exchange the two, but the opposite process (c) is not. (d) The free energy may be significantly reduced when two particles of the same species share the same potential minimum. See also Supplementary Video 2 (ESI $\dagger$ ).
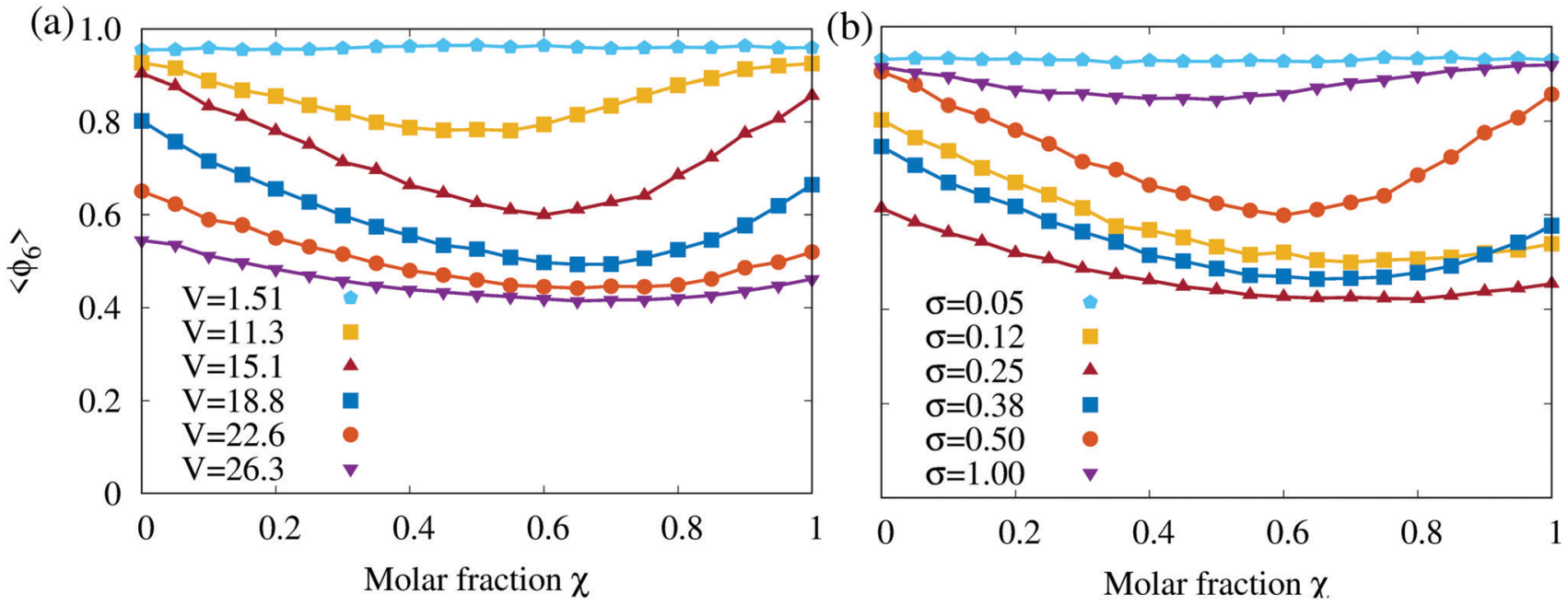

Fig. 4 Dependence of the order parameter on the speckle properties. Six-fold bond order parameter as a function of the molar fraction $(\chi)$ obtained numerically, for different values of the speckle (a) strength and (b) spatial correlation $\sigma$. Results in (a) were obtained for $\sigma=0.5$ and in (b) for $V=15.1$, and are averages over 100 samples.

underlying random optical potential. While previous studies ${ }^{19,20}$ have shown how freezing and melting are influenced by the intensity of the laser field and the particle density. We employ a disordered potential and a binary mixture where some particles interact strongly with the substrate and others are weakly interacting. This permits us to study a system where disorder and impurities are present, which is highly relevant for applications. Since the intensity of the optical forces depends on the mismatch of the indices of refraction of the particles and the surrounding medium, the particles with the larger index mismatch are more responsive (strong particles) than those with the lower mismatch (weak particles). For the parameters of the optical potential that were considered, only the strong particles respond significantly to the potential. Thus, strong particles tend to occupy the minima of the potential and nucleate structural defects in the, otherwise, periodic hexagonal structure of the weak particles. 
The density of defects is controlled by the fraction of strong particles and the statistical properties of the underlying potential. When the number of strong particles increases beyond the number of local minima that can trap them, the trapping mechanism becomes less effective and the hexagonal order is recovered as the fraction of strong particles increases.

Here, we have considered a random optical potential with Gaussian spatial correlations and a characteristic length that is of the order of the particle size. However, it is technically possible to generate other optical potentials, e.g. periodic ${ }^{27}$ or with different spatial correlations. ${ }^{30,31}$ Thus, one can control not only the density of defects but also their spatial distribution. Time-varying optical potentials or driving forces could also be employed to change the position of strong particles and defects in time, affecting the overall dynamics, what raises several relevant fundamental and applied questions. ${ }^{18,23,32,33}$ Understanding how the spatial distribution of defects influences the physical properties of materials is a question of both scientific curiosity and technological interest that can now be addressed in a systematic way.

A non-monotonic dependence of the density of defects on the particle ratio was also found for a binary mixture of Yukawa particles coupled to a random (quenched) field in ref. 34, where the particles differ in charge, which impacts the particle-particle interaction, but the response to the external field is identical. By contrast, here the particle-particle interactions are identical for both species, while their response to the external field is distinct. This difference is key to enable the external control of the density of defects, as proposed here.

\section{Materials and methods}

\section{Sample preparation}

Diluted aqueous stock solutions of polystyrene and silica colloidal spheres (microparticles $\mathrm{GmbH}$, diameter $d_{\mathrm{PS}}=6.24 \pm$ $0.22 \mu \mathrm{m}$ and $d_{\mathrm{Si}}=6.73 \pm 0.22 \mu \mathrm{m}$, respectively) were used to prepare binary solutions with different molar fractions of polystyrene particles from $\chi=0$ to $\chi=1$. The total density of particles was kept constant at $1.4 \times 10^{7} \mathrm{ml}^{-1}$. These colloidal solutions were confined in a homemade sample chamber (internal thickness $200 \mu \mathrm{m}$ ), built between a bottom glass slide (made hydrophilic by treatment in a $0.25 \mathrm{M} \mathrm{NaOH}$ solution) and a top flat-terminated fibre coupler (Thorlabs, SM1SMA) held apart by two layers of a thermoplastic spacer, which at the same time was also used for sealing the chamber. The fibre coupler was used to connect the output end of a multimode optical fiber (core diameter $105 \mu \mathrm{m}, \mathrm{NA}=0.22$, length $51 \mathrm{~m}$ ). See also Fig. 5 .

\section{Experimental setup}

A homemade inverted optical microscope setup was used for carrying out the experimental investigations of structural defects in colloidal crystals formed under random optical potentials, as schematically shown in Fig. $5 .{ }^{24}$ An image of the sample with colloidal particles was projected by a microscope objective (Nikon Plan Fluorite Imaging Objective, 20×,

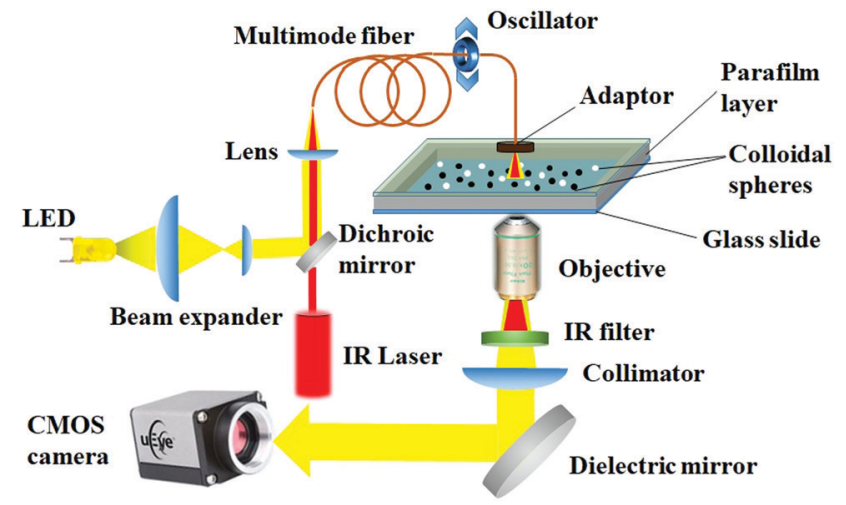

Fig. 5 Schematic representation of the experimental setup and sample chamber.

$\mathrm{NA}=0.5, \mathrm{WD}=2.1 \mathrm{~mm}$ ) onto a monochrome charge-coupled device (CCD) camera with an acquisition rate between 1 and 8 frames per second (fps). The incoherent illumination was provided by a LED lamp at $\lambda=625 \mathrm{~nm}$ coupled into the optical fiber using a dichroic mirror (Thorlabs, DMLP650). The particles were tracked by digital video microscopy. ${ }^{35}$

The static speckle light pattern with a Gaussian envelope was generated by focusing a laser beam (wavelength $\lambda=976 \mathrm{~nm}$, output power $P=90 \mathrm{~mW}$ ) into a multimode optical fiber using a plano-convex lens (focal distance $f=25.4 \mathrm{~mm}$ ). The output speckle pattern is the result of the multipath interference of the optical waves carrying random phases within the multimode optical fiber. $^{23,24,36}$ The length of the optical path between the fiber tip and the imaging plane where the colloidal particles lay (i.e., the bottom of the sample chamber) determines the final speckle grain size. The typical duration of an experiment is about 90 minutes.

The smooth optical potential was obtained by the speckle suppression using a high frequency mechanical oscillator connected to the stretched interval of the optical fiber. The vibrational frequency was adjusted with DC voltage up to $12000 \mathrm{rpm}$.

\section{Simulations}

We performed Brownian dynamics (BD) simulations of a binary mixture of $N=800$ particles with several compositions, on a two-dimensional square box with linear size $L$. The particle species differ in the strength of their response to the optical potential. The interaction potential between a pair of particles $i$ and $j$ with diameter $d_{\mathrm{p}}$ is independent of the species and is given by the repulsive part of a Lennard-Jones potential:

$$
V_{i j}(r)=\varepsilon\left[\left(\frac{d_{\mathrm{p}}}{r}\right)^{12}-\left(\frac{d_{\mathrm{p}}}{r}\right)^{6}\right],
$$

where $\varepsilon$ sets the energy scale. This is a very steep and shortranged potential that only affects neighbouring particles within a cut-off distance of $r_{\text {cut }}=2^{-1 / 6} d_{\mathrm{p}}$.

The external potential has two contributions. The first contribution is a Gaussian potential that attracts the particles 
towards the centre of the simulation box, given by

$$
V_{\text {Gaussian }}(r)= \begin{cases}-V_{\mathrm{G}_{k}} \mathrm{e}^{-\frac{(r-7.5)^{2}}{\sigma_{\mathrm{G}}{ }^{2}}} & \text { if } r>7.5 \\ 0 & \text { if } r \leq 7.5\end{cases}
$$

where $\sigma_{\mathrm{G}}$ is the width of the Gaussian and $V_{\mathrm{G}_{k}}$ sets the scale of this interaction, which depends on the particle type $k$; the interaction $V_{\mathrm{G}_{k}}$ with the most responsive (strong) particles is $2 \times$ that with the least responsive (weak) ones; $r$ is the distance to the centre of the simulation box. By definition, we ensure that the Gaussian potential is zero in the central region of the box, of radius 7.5, where we carried out the statistical analysis of the system. This potential is used to confine the particles in the centre of the box at sufficiently high densities. The second contribution to the external potential reproduces the potential generated by a speckle pattern. ${ }^{22}$ We used the Fourier filtering method (FFM) to generate numerically random potentials with Gaussian spatial correlations. ${ }^{37,38}$ The FFM takes advantage of the fact that the correlation function of a field $E(\vec{r})$, is the inverse Fourier transform of the absolute value of its Fourier coefficients, $\left|E_{\vec{k}}\right|^{2}$, as stated by the Wiener-Khinchin theorem. ${ }^{39}$ This relation allows us to sample random Fourier coefficients that when transformed back into real space describe a random potential with the desired spatial correlations. The depths of those potentials have a Gaussian distribution. To convert it into an exponential distribution, as measured for the speckle, we used the following procedure: the random surface is discretized in $1024 \times 1024$ cells, which we sort by the intensity of the potential. Then, we produce a sorted list of intensities drawn from an exponential distribution and substitute each cell intensity by the corresponding entry on the ranked list of intensities. We tested this procedure with Gaussian and power-law correlation functions and confirmed that it does lead to the desired distribution of intensities, without affecting the nature of the correlation function. The forces due to this potential are then calculated using finite differences. In all simulations, we considered Gaussian correlations with a dispersion $\sigma$. When $\sigma<d_{\mathrm{p}}$ (where $d_{\mathrm{p}}$ is the diameter of the particles) the speckle features vary on distances shorter than the particle size and we need to consider an effective speckle pattern that is the result of the integration of the speckle intensities over the particle volume (see below section "Effective speckle properties"). The results present in Fig. 1 and 2 were achieved with $\sigma=0.4$. The potentials strength ratio is $V_{\mathrm{G}} / V=1$ in the simulations presented in Fig. 1, 2, 4(a) and (b).

The motion of a particle $i$ in the surrounding medium is described by the overdamped Langevin equation

$$
\gamma \frac{\mathrm{d} \vec{r}_{i}}{\mathrm{~d} t}=-\vec{\nabla}_{i}\left[\sum_{j} V_{i j}(r)+V_{\text {ext }}\left(\vec{r}_{i}\right)\right]+\vec{\xi}_{i}, \quad j \neq i,
$$

where $\gamma$ is the Stokes-Einstein friction coefficient and $\vec{\xi}_{i}$ is a random stochastic term that mimics the thermal noise that results from the interaction with the medium. This term is given by a normal distribution with zero mean and auto-correlation that is independent of space and time and proportional to the thermostat temperature T, i.e. $\left\langle\xi_{i}^{n}(t) \xi_{i}^{1}\left(t^{\prime}\right)\right\rangle=2 k_{\mathrm{B}} T \gamma_{i} \delta_{\mathrm{nl}} \delta\left(t-t^{\prime}\right)$, where $n$ and $l$ are indices that run over the space dimensions and $k_{\mathrm{B}}$ is the Boltzmann constant. The characteristic time is defined as $\tau=d_{\mathrm{p}}{ }^{2} \gamma / k_{\mathrm{B}} T$. Eqn (4) is integrated following the algorithm developed by Branka and Heyes, ${ }^{40}$ i.e. a second-order stochastic Runge-Kutta scheme, with a time step of $\Delta t=10^{-4} \tau$. We set the diameter of the particle, $d_{\mathrm{p}}$, as the unit length, the simulation box has linear size $L=50$ and the width of the external Gaussian potential is $\sigma_{\mathrm{G}}=L / 2$. The energy is given in units of $k_{\mathrm{B}} T$ with $\varepsilon=10$ and $V_{\mathrm{G}}=200$. The simulations were run for $2 \times 10^{4} \tau$ and the data used in the calculations was taken in the last $1.5 \times 10^{3} \tau$, when the evolution was found to be in the stationary state in the centre of the box. For all data points, we used 100 samples to average the relevant quantities. While we do not expect a strong dependence on the geometry of the experimental setup, in order to make a direct comparison with the experimental results, rather than using periodic boundary conditions, we considered the same circular confinement with an external potential. This also allows us to study the initial dynamics that result from increasing the local concentration in the center due to the confining potential.

\section{Conflicts of interest}

There are no conflicts to declare.

\section{Acknowledgements}

André S. Nunes, Margarida M. Telo da Gama and Nuno A. M. Araújo acknowledge financial support from the Portuguese Foundation for Science and Technology (FCT) under Contracts no. EXCL/FIS-NAN/0083/2012, UIDB/00618/2020, UIDP/00618/ 2020, SFRH/BD/119240/2016 and PTDC/FIS-MAC/28146/2017 (LISBOA-01-0145-FEDER-028146). Margarida M. Telo da Gama and Nuno Araújo would like to thank the Isaac Newton Institute for Mathematical Sciences for support and hospitality during the program "The mathematical design of new materials" where the final version of this manuscript was completed. This program was supported by EPSRC Grant Number: EP/R014604/1. Giorgio Volpe acknowledges support from the Royal Society under grant RG150514. Iryna Kasianiuk acknowledges partial support of Tübitak grant 115F401. Denis Kasyanyuk acknowledges partial support of Tübitak grant 116F111. Agnese Callegari acknowledges partial support of Tübitak grant 115F401 and 116F111. We also acknowledge Parviz Elahi for his help with the experimental setup.

\section{References}

$1 \mathrm{~W}$. Bollmann, Crystal defects and crystalline interfaces, Springer Science \& Business Media, 2012.

2 K. Pyka, J. Keller, H. L. Partner, R. Nigmatullin, T. Burgermeister, D. M. Meier, K. Kuhlmann, A. Retzker, M. B. Plenio, W. H. Zurek, A. del Campo and T. E. Mehlstäubler, Topological defect formation and spontaneous symmetry breaking in ion coulomb crystals, Nat. Commun., 2013, 4, 2291.

3 W. H. Zurek, Causality in condensates: Gray solitons as relics of BEC formation, Phys. Rev. Lett., 2009, 102, 105702. 
4 A. del Campo, G. De Chiara, G. Morigi, M. B. Plenio and A. Retzker, Structural defects in ion chains by quenching the external potential: The inhomogeneous kibble-zurek mechanism, Phys. Rev. Lett., 2010, 105, 075701.

5 I. M. Lifshitz and A. M. Kosevich, The dynamics of a crystal lattice with defects, Rep. Prog. Phys., 1966, 29, 217.

6 D. T. J. Hurle and P. Rudolph, A brief history of defect formation, segregation, faceting, and twinning in meltgrown semiconductors, J. Cryst. Growth, 2004, 264, 550.

7 K. Chen, R. Kapadia, A. Harker, S. Desai, J. S. Kang, S. Chuang, M. Tosun, C. M. Sutter-Fella, M. Tsang, Y. Zeng, D. Kiriya, J. Hazra, S. R. Madhvapathy, M. Hettick, Y.-Z. Chen, J. Mastandrea, M. Amani, S. Cabrini, Y.-L. Chueh, J. W. Ager III, D. C. Chrzan and A. Javey, Direct growth of single-crystalline III-V semiconductors on amorphous substrates, Nat. Commun., 2016, 7, 10502.

8 T. Boeck, F. Ringleb and R. Bansen, Growth of crystalline semiconductor structures on amorphous substrates for photovoltaic applications, Cryst. Res. Technol., 2017, 52, 1600239.

9 M. Heyde, Structure and motion of a 2D glass, Science, 2013, 342, 201.

10 M. S. Kulkarni, A selective review of the quantification of defect dynamics in growing Czochralski silicon crystals, Ind. Eng. Chem. Res., 2005, 44, 6246.

11 M. J. Kramer, M. I. Mendelev and R. E. Napolitano, In situ observation of antisite defect formation during crystal growth, Phys. Rev. Lett., 2010, 105, 245501.

12 N. Faleev, N. Sustersic, N. Bhargava, J. Kolodzey, S. Magonov, D. J. Smith and C. Honsberg, Structural investigations of SiGe epitaxial layers grown by molecular beam epitaxy on Si (001) and Ge (001) substrates: Ii - Transmission electron microscopy and atomic force microscopy, J. Cryst. Growth, 2013, 365, 35.

13 S. Wang, A. Robertson and J. H. Warner, Atomic structure of defects and dopants in 2 d layered transition metal dichalcogenides, Chem. Soc. Rev., 2018, 47, 6764.

14 S. Deutschländer, P. Dillmann, G. Maret and P. Keim, Kibble-zurek mechanism in colloidal monolayers, Proc. Natl. Acad. Sci. U. S. A., 2015, 112, 6925.

15 W. T. M. Irvine, M. J. Bowick and P. M. Chaikin, Fractionalization of interstitials in curved colloidal crystals, Nat. Mater., 2012, 11, 948.

16 A. S. Nunes, N. A. M. Araújo and M. M. Telo da Gama, Selfassembly of colloidal bands driven by a periodic external field, J. Chem. Phys., 2016, 144, 034902.

17 A. T. Pham, R. Seto, J. Schönke, D. Y. Joh, A. Chilkoti, E. Fried and B. B. Yellen, Crystallization kinetics of binary colloidal monolayers, Soft Matter, 2016, 12, 7735.

18 T. Brazda, C. July and C. Bechinger, Experimental observation of Shapiro-steps in colloidal monolayers driven across timedependent substrate potentials, Soft Matter, 2017, 13, 4024.

19 C. Bechinger, M. Brunner and P. Leiderer, Phase behavior of two-dimensional colloidal systems in the presence of periodic light fields, Phys. Rev. Lett., 2001, 86, 930-933.

20 A. Chowdhury, B. J. Ackerson and N. A. Clark, Laser-induced freezing, Phys. Rev. Lett., 1985, 55, 833-836.
21 See ESI $\dagger$ at [url].

22 G. Volpe, G. Volpe and S. Gigan, Brownian motion in a speckle light field: tunable anomalous diffusion and selective optical manipulation, Sci. Rep., 2014, 4, 3936.

23 G. Volpe, L. Kurz, A. Callegari, G. Volpe and S. Gigan, Speckle optical tweezers: Micromanipulation with random light fields, Opt. Express, 2014, 22, 18159.

24 E. Pinçe, S. K. Velu, A. Callegari, P. Elahi, S. Gigan, G. Volpe and G. Volpe, Disorder-mediated crowd control in an active matter system, Nat. Commun., 2016, 7, 10907.

25 T. L. Alexander, J. E. Harvey and A. R. Weeks, Average speckle size as a function of intensity threshold level: comparison of experimental measurements with theory, Appl. Opt., 1994, 33, 8240-8250.

$26 \mathrm{H}$. Lin and P. Yu, Speckle mechanism in holographic optical imaging, Opt. Express, 2007, 15, 16322-16327.

27 P. H. Jones, O. M. Maragò and G. Volpe, Optical tweezers: Principles and applications, Cambridge University Press, Cambridge, UK, 2015.

28 L. P. García, J. D. Pérez, G. Volpe, A. V. Arzola and G. Volpe, High-performance reconstruction of microscopic force fields from Brownian trajectories, Nat. Commun., 2018, 9, 5166.

29 A. S. Nunes, A. Gupta, N. A. M. Araújo and M. M. Telo da Gama, Field-driven dynamical demixing of binary mixtures, Mol. Phys., 2018, 116, 3224.

30 Y. Bromberg and H. Cao, Generating non-rayleigh speckles with tailored intensity statistics, Phys. Rev. Lett., 2014, 112, 213904.

31 N. Bender, H. Yilmaz, Y. Bromberg and H. Cao, Customizing speckle intensity statistics, Optica, 2018, 5, 595.

32 D. G. Grier, A revolution in optical manipulation, Nature, 2003, 424, 810.

33 C. Reichhardt and C. J. O. Reichhardt, Depinning and nonequilibrium dynamic phases of particle assemblies driven over random and ordered substrates: a review, Rep. Prog. Phys., 2017, 80, 026501.

34 C. Reichhardt and C. J. O. Reichhardt, Disordering transitions and peak effect in polydispersity particle systems, Phys. Rev. E: Stat., Nonlinear, Soft Matter Phys., 2008, 77, 041401.

35 J. C. Crocker and D. G. Grier, Methods of digital video microscopy for colloidal studies, J. Colloid Interface Sci., 1996, 179, 298.

36 A. P. Mosk, A. Lagendijk, G. Lerosey and M. Fink, Controlling waves in space and time for imaging and focusing in complex media, Nat. Photonics, 2012, 6, 283.

37 H. A. Makse, S. Havlin, M. Schwartz and H. E. Stanley, Method for generating long-range correlations for large systems, Phys. Rev. E: Stat. Phys., Plasmas, Fluids, Relat. Interdiscip. Top., 1996, 53, 5445.

38 E. A. Oliveira, K. J. Schrenk, N. A. M. Araújo, H. J. Herrmann and J. S. Andrade, Optimal-path cracks in correlated and uncorrelated lattices, Phys. Rev. E: Stat., Nonlinear, Soft Matter Phys., 2011, 83, 046113.

39 The Science of Fractal Images, ed. H.-O. Peitgen and D. Saupe, Springer-Verlag New York, Inc., New York, NY, USA, 1988.

40 A. C. Brańka and D. M. Heyes, Algorithms for brownian dynamics computer simulations: Multivariable case, Phys. Rev. E: Stat. Phys., Plasmas, Fluids, Relat. Interdiscip. Top., 1999, 60, 2381. 